\title{
Atomic time scales TAI and TT(BIPM): present performances and prospects
}

\author{
Gérard Petit \\ Bureau International des Poids et Mesures \\ 92312 Sèvres Cedex, France \\ email: gpetit@bipm.org
}

\begin{abstract}
We review the stability and accuracy achieved by the reference atomic time scales TAI and TT(BIPM). We show that they presently are at the level of a few $10^{-16}$ in relative value, based on the performance of primary standards, of the ensemble time scale and of the time transfer techniques. We consider how the $1 \times 10^{-16}$ value could be reached or superseded and which are the present limitations to attain this goal.
\end{abstract}

Keywords. Time, Reference systems

\section{Introduction}

International Atomic Time TAI gets its stability from some 350 atomic clocks worldwide that generate the free atomic scale EAL and its accuracy from a small number of primary frequency standards (PFS) which are used to steer the EAL frequency. Terrestrial Time is a coordinate time in the geocentric reference system defined by the International Astronomical Union. TAI provides one realization of TT but it is not optimal because of operational constraints. The BIPM therefore computes in deferred time another realization, TT(BIPM), which is based on a weighted average of the evaluations of TAI frequency by the PFS. A new version is computed each January, the latest available being TT(BIPM08) available at ftp://tai.bipm.org/TFG/TT(BIPM).

We review the stability and accuracy achieved by the reference atomic time scales TAI and TT(BIPM). We show that they presently are at the level of a few $10^{-16}$ in relative value, based on the performance of primary standards, of the ensemble time scale and of the time transfer techniques. We consider how the $1 \times 10^{-16}$ value could be reached or superseded and which are the present limitations to attain this goal.

\section{Achieving sub- $10^{-15}$ accuracy}

The stability of atomic time scales, the PFS accuracy and the capabilities of frequency transfer all achieve about a few parts in $10^{16}$ in relative frequency stability.

The stability and accuracy of time scales has been extensively studied by Petit (2007). The 1-month stability is that of EAL, at $3-4 \times 10^{-16}$. The estimated accuracy of $\mathrm{TT}(\mathrm{BIPM})$ over recent yers is close to $5 \times 10^{-16}$ or below. This is due to the ever increasing number of Cs fountain evaluations (about 170 since 1999, 45 of them in 2008), and to the improved accuracy of each evaluation. Using TT(BIPM08) as a reference shows that EAL is affected by a significant drift and that the long-term instability of TAI is between $1 \times 10^{-15}$ and $1 \times 10^{-15}$, a factor two or three worse than TT(BIPM).

The time transfer techniques presently used in TAI are Global Positioning System (GPS) code measurements in a mode called all in view, which produces best results when using dual-frequency receivers, and two-way time transfer (TW) using telecommunication 
satellites, and the implementation of GPS phase and code measurements in TAI is under way (Petit 2009). It has been shown, see e.g. Bauch et al. (2006), that these techniques are capable of a frequency transfer uncertainty of $1 \times 10^{-15}$ in one day (GPS code and phase, TW at short distance) or in a few days (GPS code only, TW at long distance). For TAI, where the optimal stability is searched at 1-month averaging, the contribution of frequency transfer to the instability is expected to be a few parts in $10^{16}$.

\section{Reaching $1 \times 10^{-16}$ and beyond}

¿From numerous recent publications, see e.g. in parts II, IV and V of Maleki (2009), it is clear that some frequency standards have reached a level where all systematic effects may be estimated with an uncertainty at $1 \times 10^{-16}$ or below, and the number of potential candidates in this group is expected to increase continuously. Therefore PFS will have the capacity to reach the level of $1 \times 10^{-16}$ and to surpass it.

Progresses in the ensemble scale EAL have been due to an increase in the number of clocks and to changes in the algorithm. Some progress can still be expected but it is unlikely that this can lead to 1 -month instability of $1 \times 10^{-16}$, as this would require a 10 -fold increase in the number of clocks used. We can expect a new generation of stable clocks, with a stability performance in the low $10^{-16}$ at 1 -month averaging time and seamless continuous operation for long periods. Possible candidates may be emerging, see e.g. pp 308 and 321 in Maleki (2009).

The techniques presently used for time transfer may progress, thanks to new or modernized navigation systems, or by using more data points for TW, or by mixing the two techniques (Jiang \& Petit 2009). Ultimately, however, these techniques will find their limitations. Several new techniques have emerged that should be able to to provide frequency transfer uncertainty in the low $10^{-17}$ region and possibly below. One is based on a microwave link to a low Earth orbit payload with one uplink and two downlink signals (Seidel et al. 2008) and another one (T2L2) on a two-way optical link to a low Earth orbit payload (Samain et al. 2008). Both promise a frequency uncertainty below $1 \times 10^{-16}$ at 1 -day averaging, if the clock of the space payload is stable enough. Another technique, even more performant although limited in spatial extension, transfers a stable laser frequency over a standard fiber link (Lopez et al. 2008).

\section{Conclusions}

We have shown that the present performance of the reference atomic time scales TAI and $\mathrm{TT}(\mathrm{BIPM})$ is in the region of a few parts in $10^{16}$ in stability and accuracy. Improving to $1 \times 10^{-16}$ and below is not out of reach but may require the development of a new generation of commercial atomic clocks or a new approach to the problem of elaborating an ensemble time scale, or both.

\section{References}

Bauch, A., Achkar, J., Bize, S. et al. 2006, Metrologia, 43, 109-120

Jiang, Z. \& Petit, G. 2009, Metrologia, 46-3, 305-314

Lopez, X., Amy-Klein, A., \& Daussy, C. 2008, Eur. Phys. J., D 48, 35-41

Maleki L. (ed.) 2009, Proc $7^{\text {th }}$ Symp. Freq. Standards and Metrology (World Scientific), 308-313

Petit, G. 2007, Proc. 21 ${ }^{\text {st }}$ EFTF conference, 391-394

Petit, G. 2009, Proc. EFTF-IFCS joint conference, 116-119

Samain, E., Weick, J., Vrancken, P. et al. 2008, Int. J. Mod. Phys., D 17-7, 1043

Seidel, A., Hess, M. P., Kehrer, J. et al. 2008, Proc. $22^{\text {nd }}$ EFTF conference 